\title{
Album Militaire
}

CAVALERIE

Service Intérieur

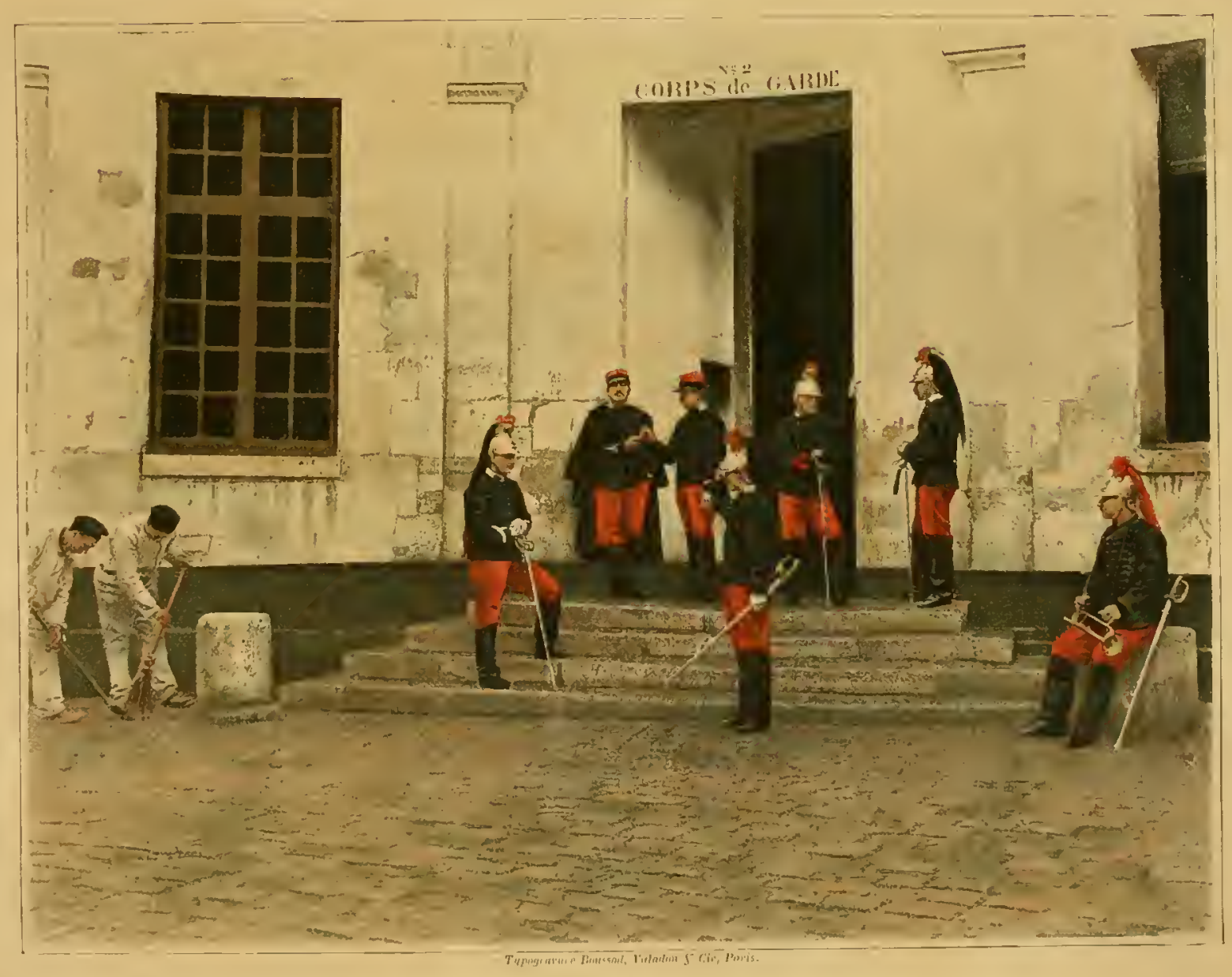

QCIBTHE HE CATLERE 


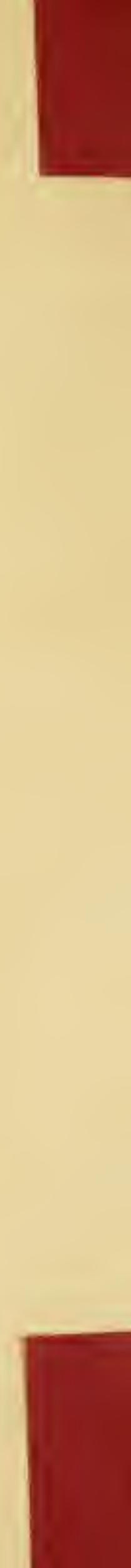


GA VA L ER I E

Service Intèrieur

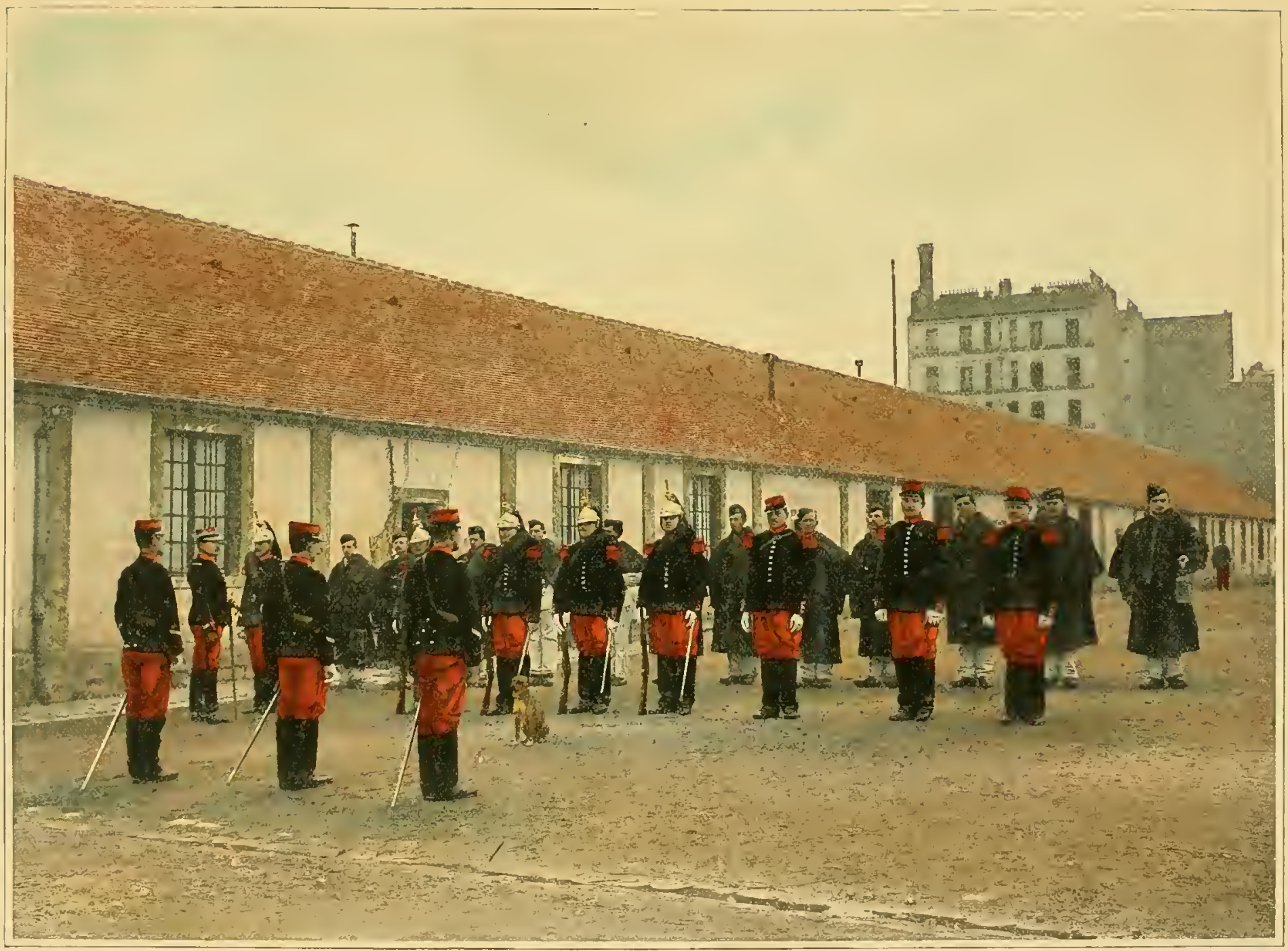

LA PARADE

A l'heure fixée par les ordres de la Place, la garde montante, composée des gradés et cavaliers qui doivent fournir les différents postes et la garde de police, est réunie dans la cour du quartier et inspectée par le capitaine de semaine qui la fait ensuite défiler devant lui au commandement de ladjudant de semaine.

Les gardes d'ícurie, en tenue de corvée, sont réunis de mème par le maréchal des logis de semaine de chaque escadron. 
GAVALER I E

Service Intérieur

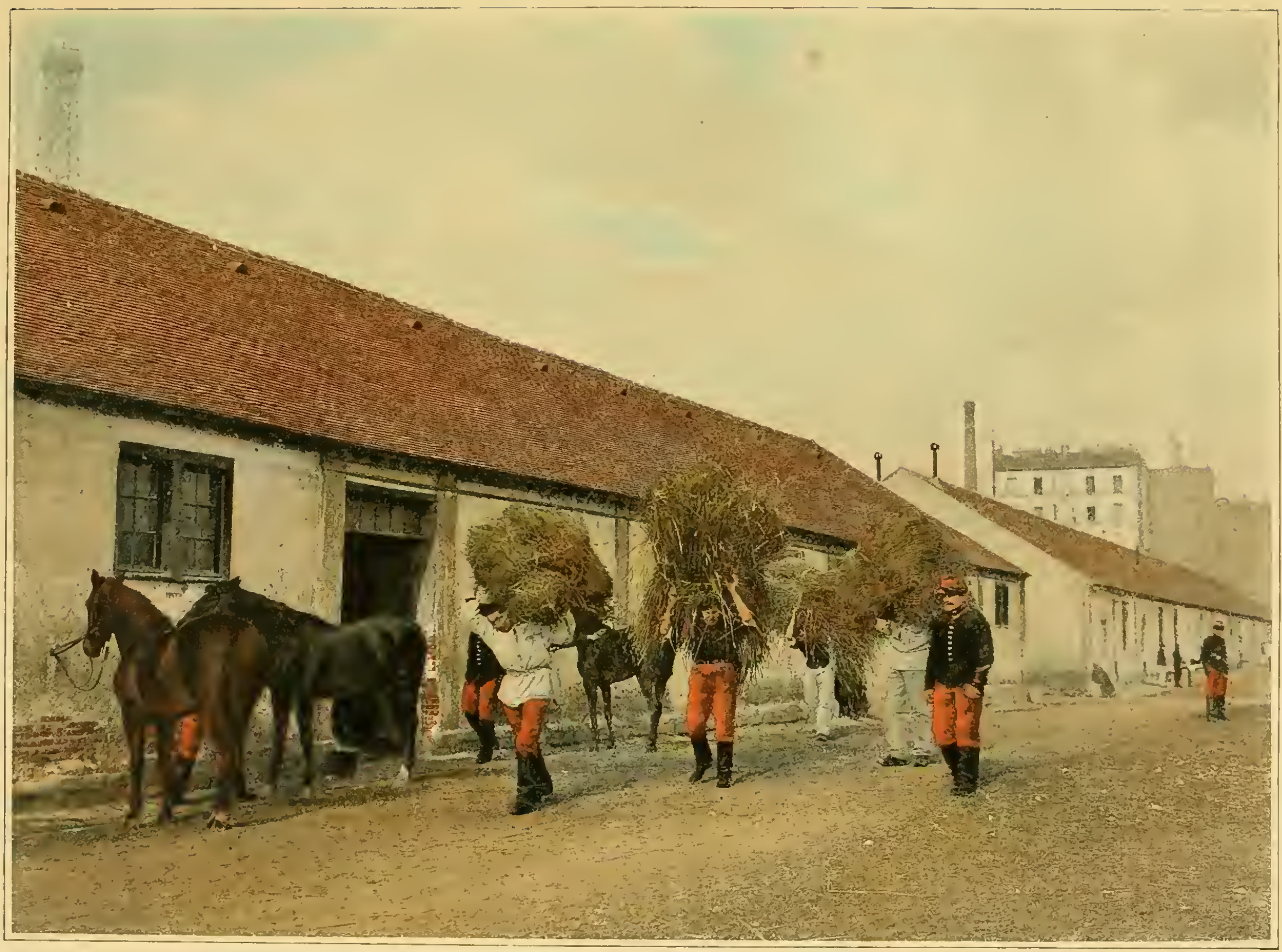

LA GORVÉE DE FOURRAGES

Quand arrive l'heure du repas des chevaux, les gardes d'écurie, sous la conduite du maréchal des logis de semaine, vont chercher au magasin de l'escadron le fourrage nécessaire pour donner "la bolte à coco ". 
CAVA LERIE

Service Intérieur

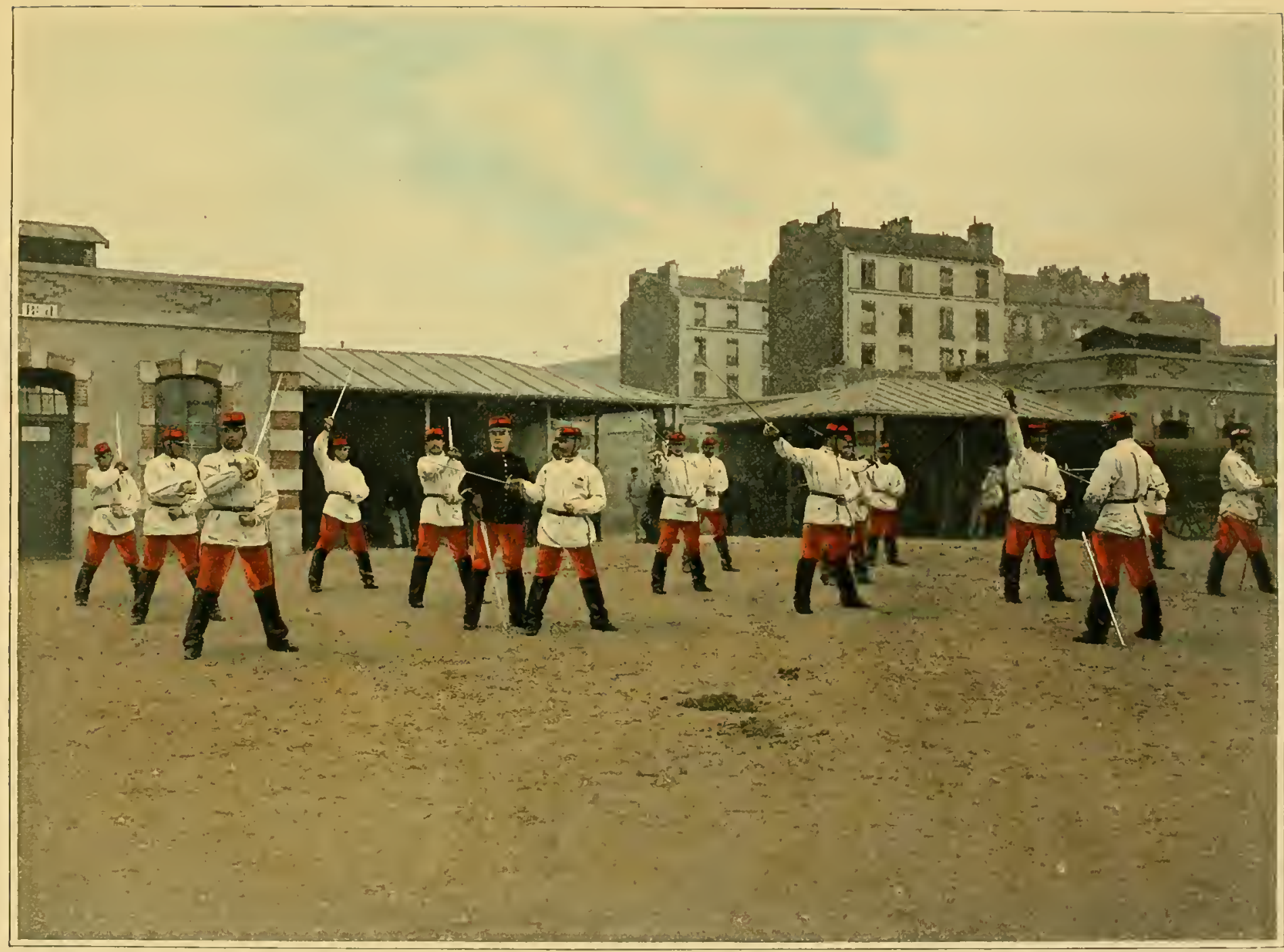

ÉCOLE DU GAVALIER A PIED - MANIEMENT DU SABRE

Avant d'exercer les cavalicrs au maniement du sabre a cheval. on leur donne, pour plus de facilité, cette instruction à pied, tout en leur faisant prendre une position se rapprocliant de celle quils auront, quand ils enfourcherom leur monturc, les rênes tenues de la main gauche. 


\section{CIVALERIE}

Service Intérieur

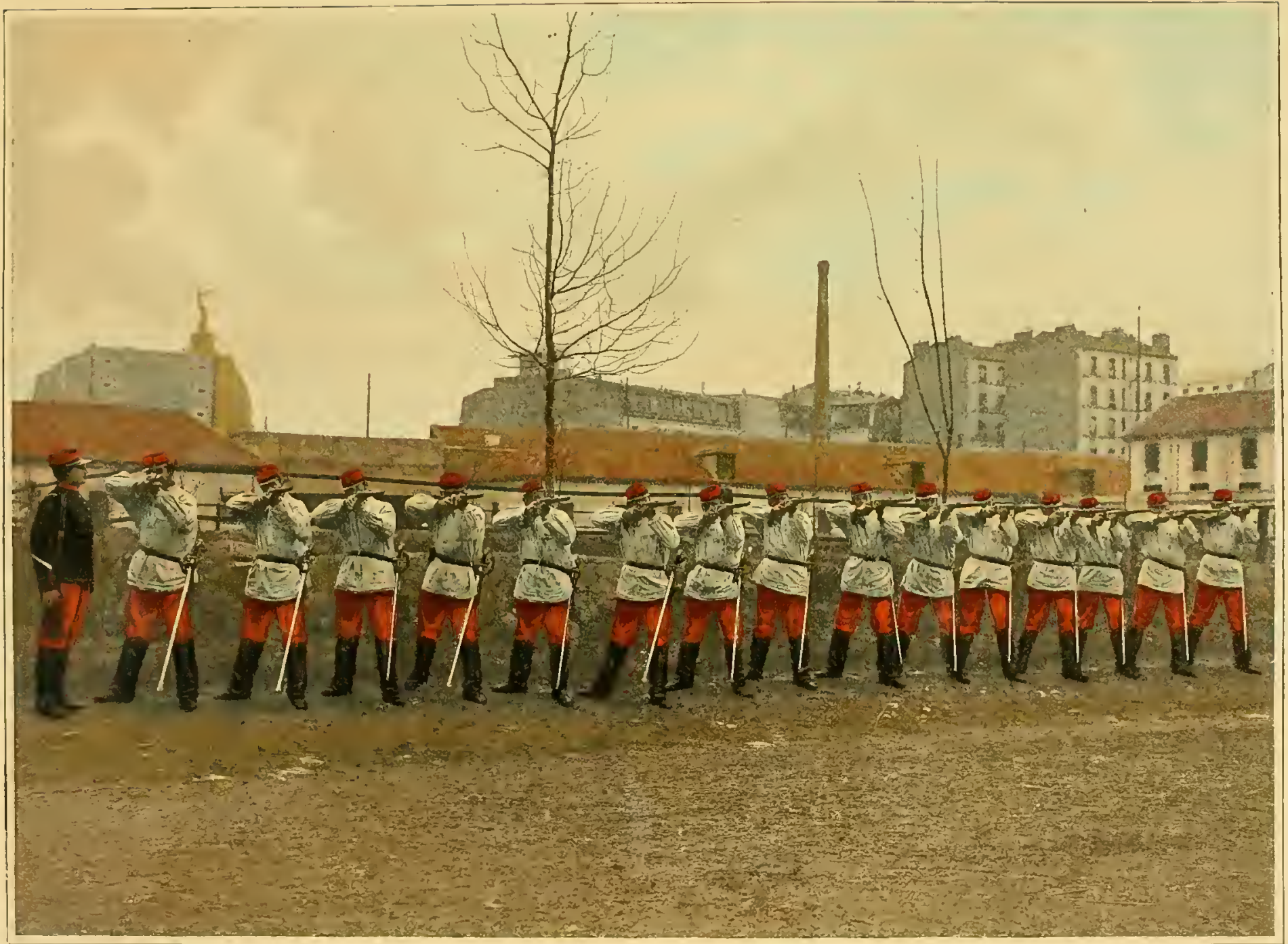

ÉGOLE DU CAVALIER A PIED - MANIEMENT DE LA GARABine

Tous les régiments de cavalerie sont armés de la carabine et recoivent l'instruction du " combat à pied », Dans maintes circonstances le cavalier peut ètre appelé à se servir utilement de cette arme, aussi les excrcices de tir sont ils soigneusement exécutés. 
GA VAISERE

Service Intèrieur

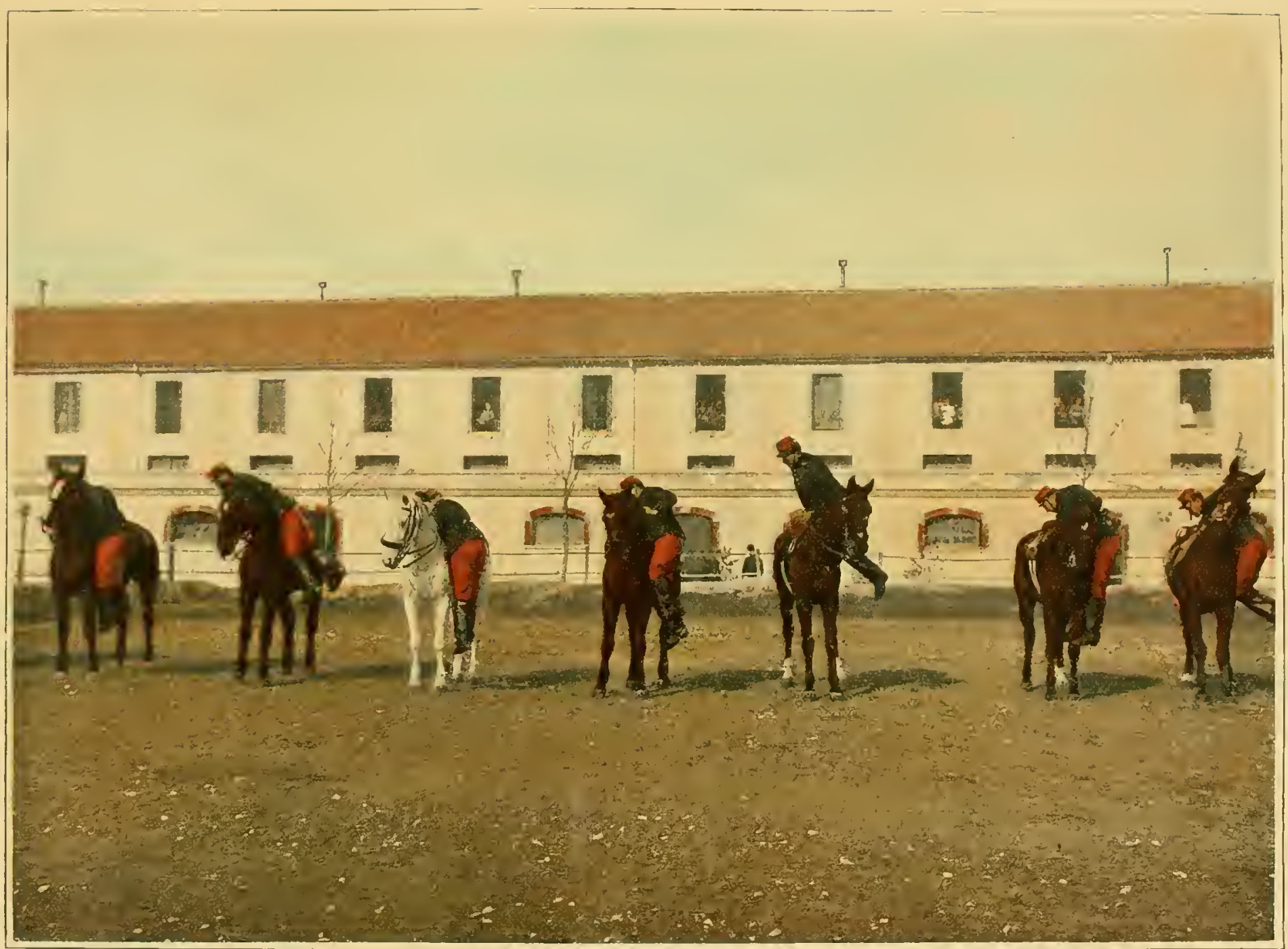

ÉCOLE DU GAVALIER A GHEVAL - SAUTER A TERRE ET A GHEVAL

Pour sauter à cheval, le cavalier prend une poignée de crins dans la main gauche, flace la main droite sur le pommeau, fait une battuc, et hop..... c'est là qu'il est bon de ne pas trop respecter les lois de la pesanteur. 
GA VA L ERIE

Service Intérieur

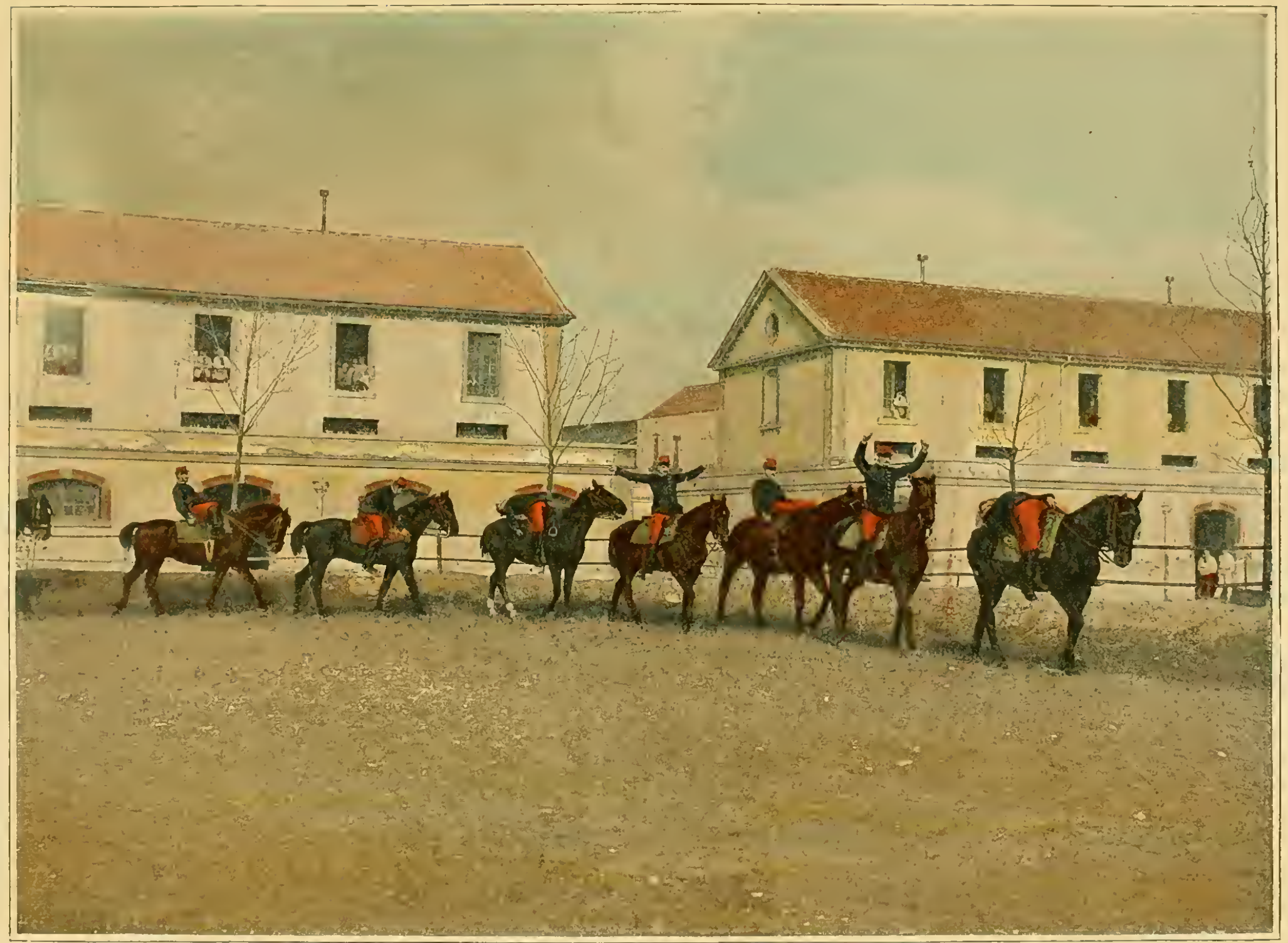

ÉGOLE DU GAVALIER A GHEVAL - EXERGIGES D'ASSOUPLISSEMENT

Les cxercices d'assouplissement constituent la gymnastique du cavalier. Chacun d'eux a un but déterminé, correspondant à un manque de souplesse ou à un défaut de conformation. C'est à l'instructeur qu'il apparticnt de prescrire a chacun d'exécuter l'assouplissement ou les assouplissements dont il a besoin. 
CA VALERIE

Service Intérieur

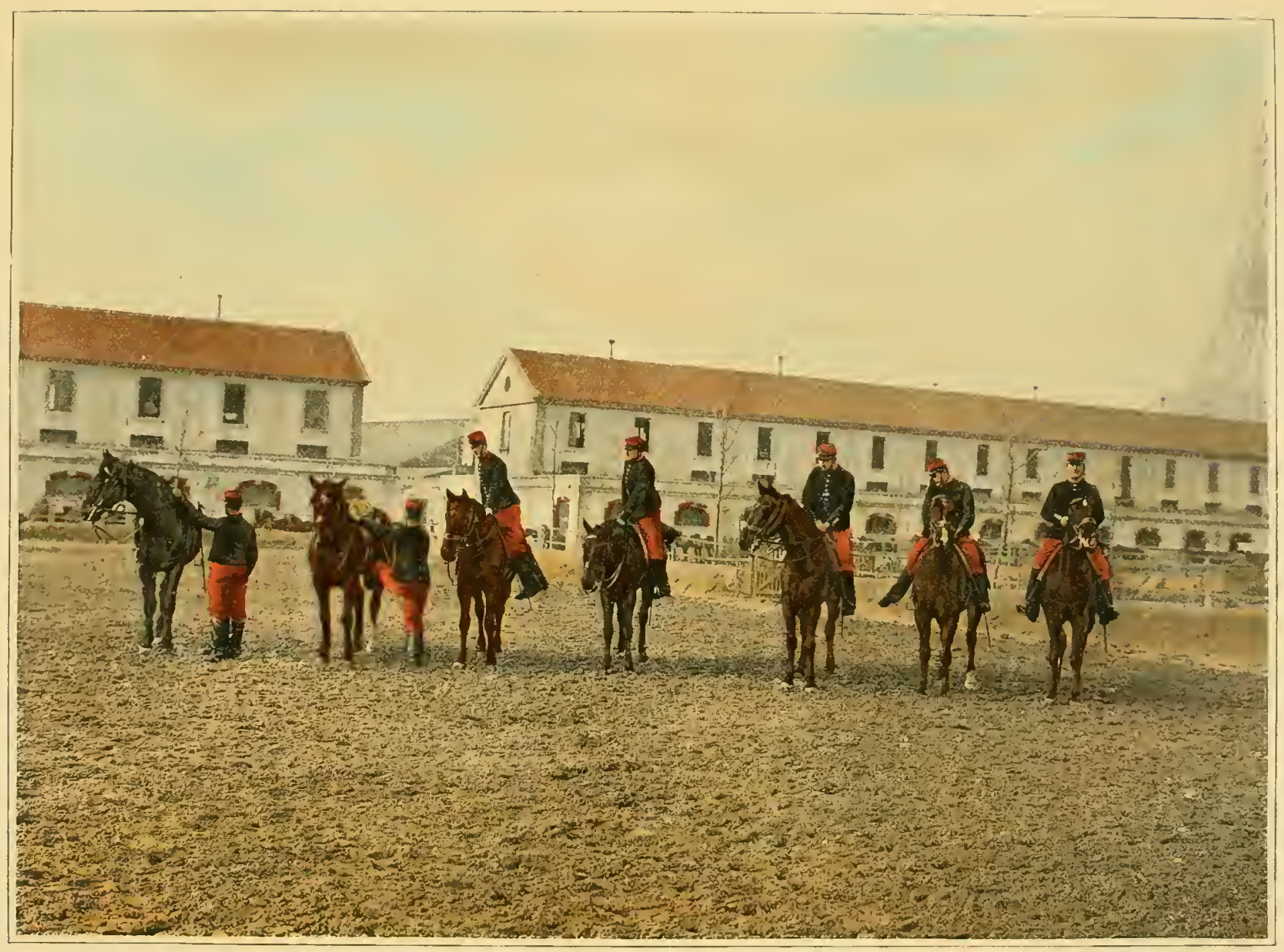

ÉCOLE DU GAVALIER A GHEVAL - MONTER A GHEVAL ET METTRE PIED A TERRE

Quand le cavalier commence à avoir sultisamment d'assiette, on lui apprend à se servir des étriers. Une des premières leçons consiste à lexercer à "monter à cheval ". 


\section{G A V A L E R I E}

Service Intérieur

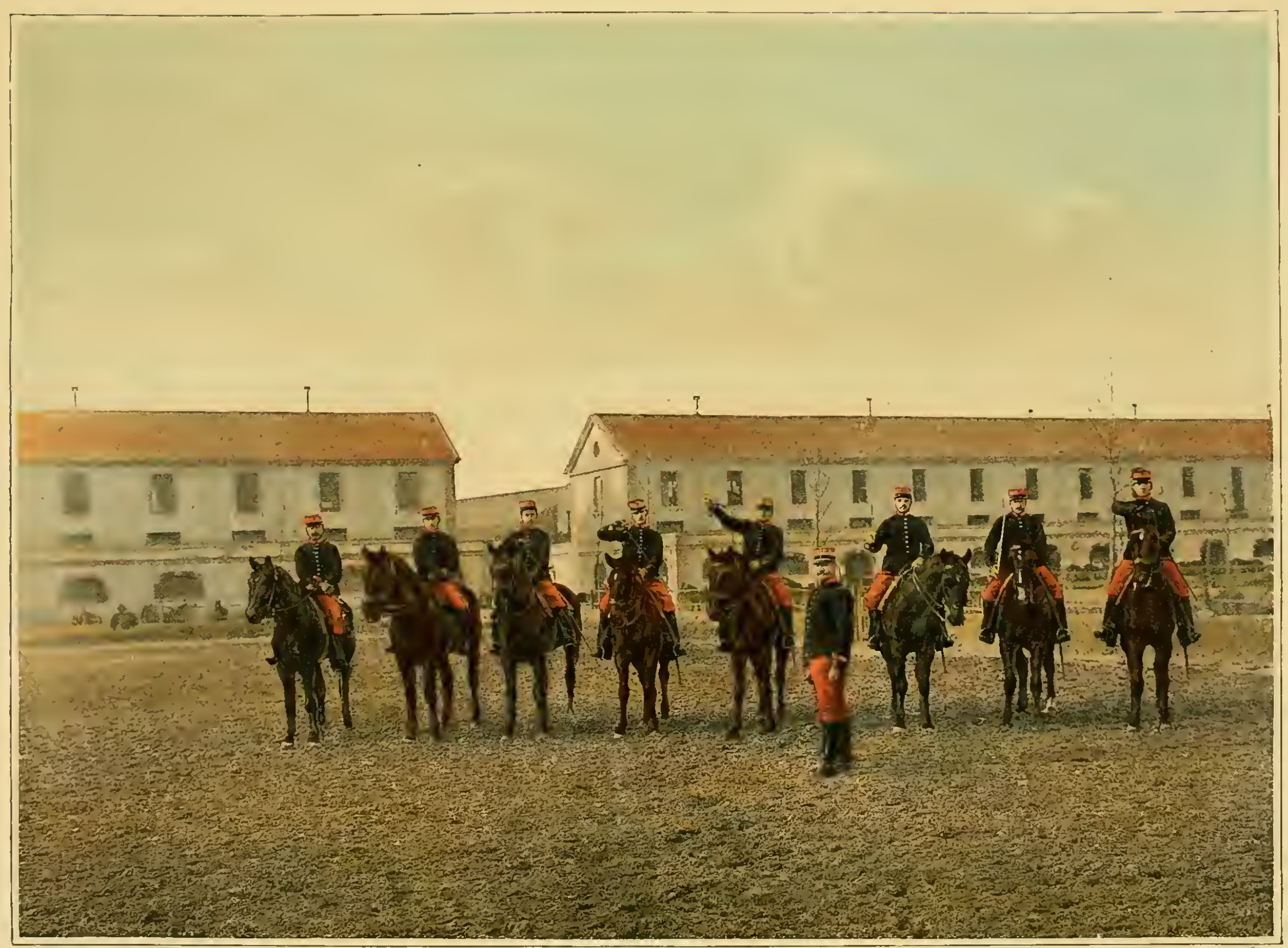

ÉGOLE DU GAVALIER A GHEVAL - MANIEMENT DU SABRE

Le sabre est l'arme, par excellence, du cavalier. Aussi est-il trẻs important que les hommes de recrue apprennent à le manier aisément. On commence par les exercer à mettre le sabre à la main et à le remettre au fourreau. 


\section{GAVILERII}

\section{Service Intérieur}

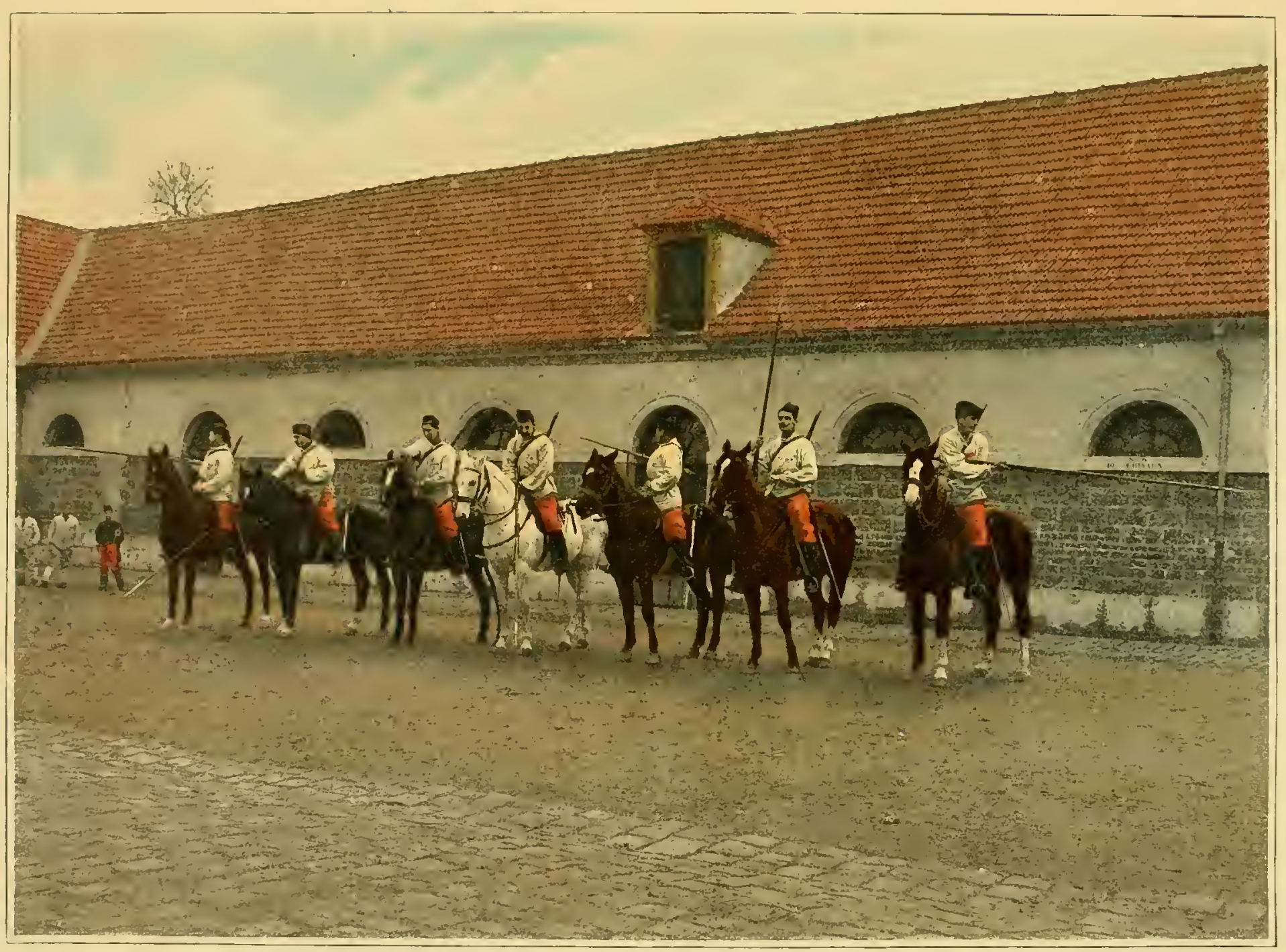

ÉCOLE DU CAVALIER A GHEVAL - EXERGice DE LA LANGE

Douze régiments de dragons sont armés de la lance, et les jeunes soldats qui y sont incorporés doivent cn apprendre le maniement, en même temps que celui du sabre et de la carabine. L'emploi de cette arme demande des cavaliers habiles et bien en selle. 
( I I A LERIE

Service Intėrieur

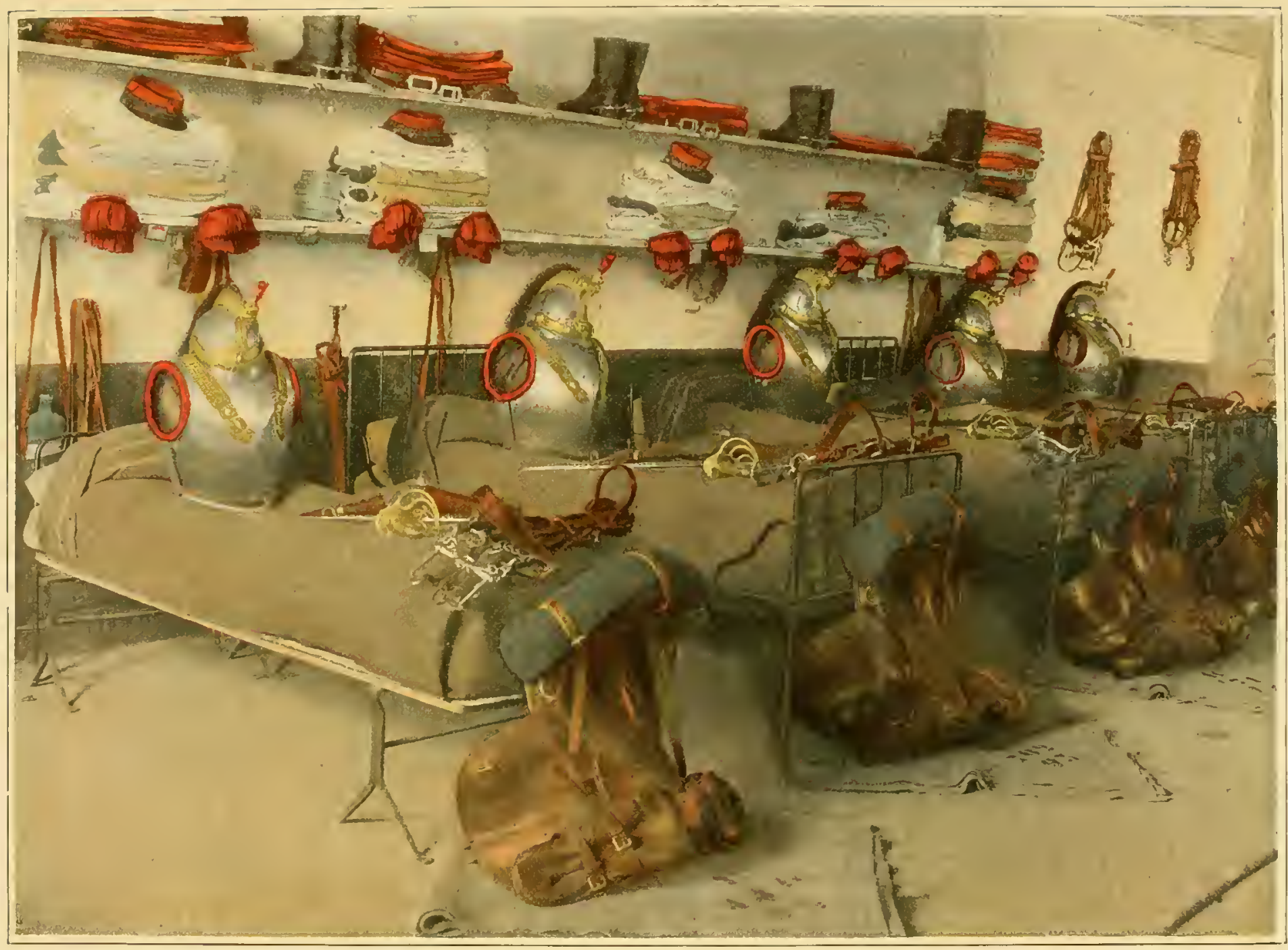

REVUE D'ARMES ET DE HARNAGHEMENT

Un aprés-midi par semaine est généralement consacré a la rcvue de l'habillement. du felit équjpement, de l'armement ou du harnachement de l'escadron.

Les effets sont disposés sur les lits. Un bon cavalier tient à honncur de présenter toujours les siens aussi propres que possible; quant aux paresseux, le brigadier d'cscouade est lá pour les faire "astiquer ". 
C.IVILERIE

Service Intérieur

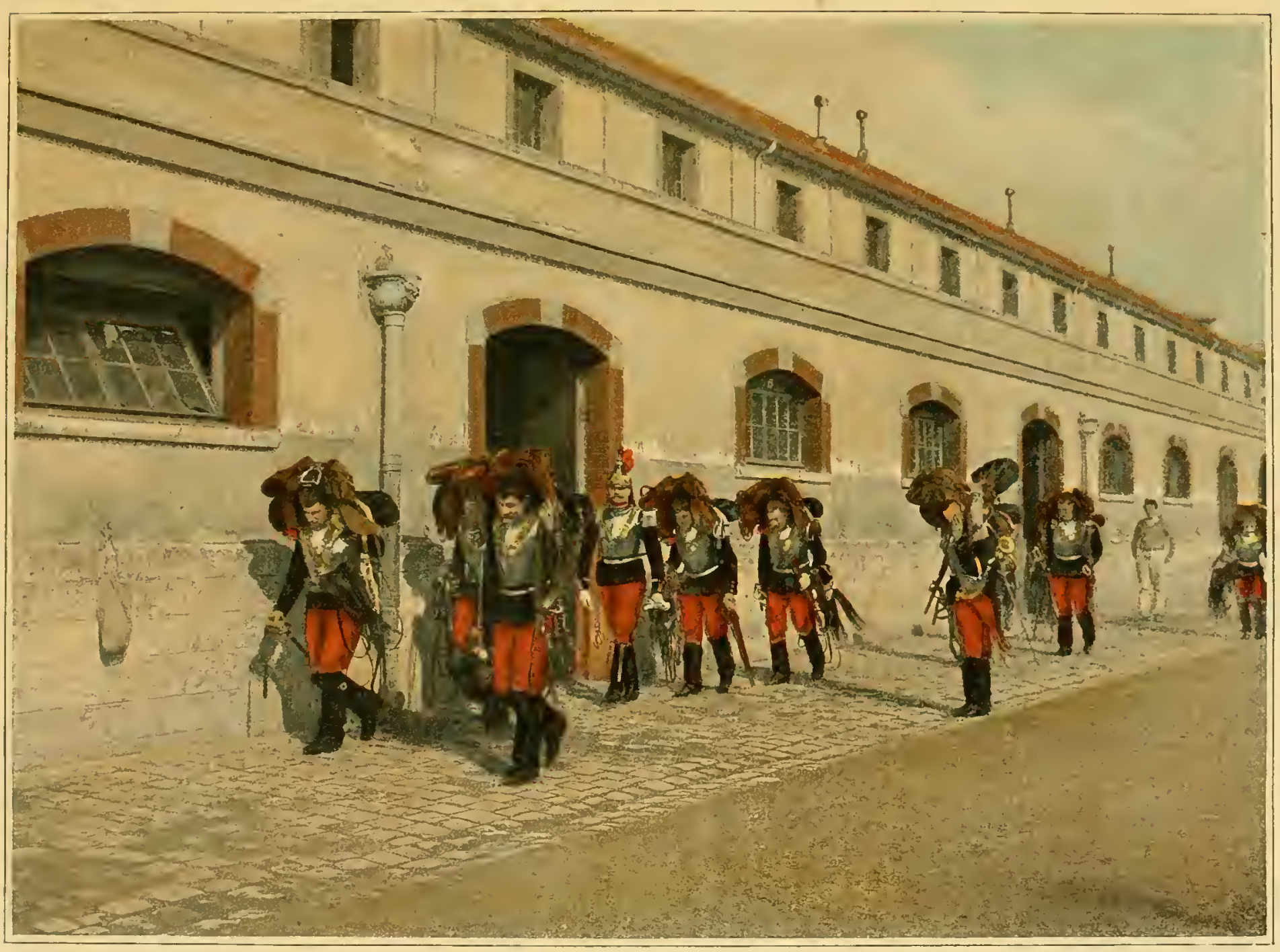

A LA DESGENTE DE GHEVAL

Dès le retour au quartier. on commence par décharger les chevaux, on monte les paquetages dans les chambres, on y laisse les armes, puis on redescend donner un coup de bouchon, faire boire et distribuer l'avoine. Le cavalier ne songe à lui-mème qu’après avoir soigné sa monture. 
GAVALERIE

Service In tẻrieur

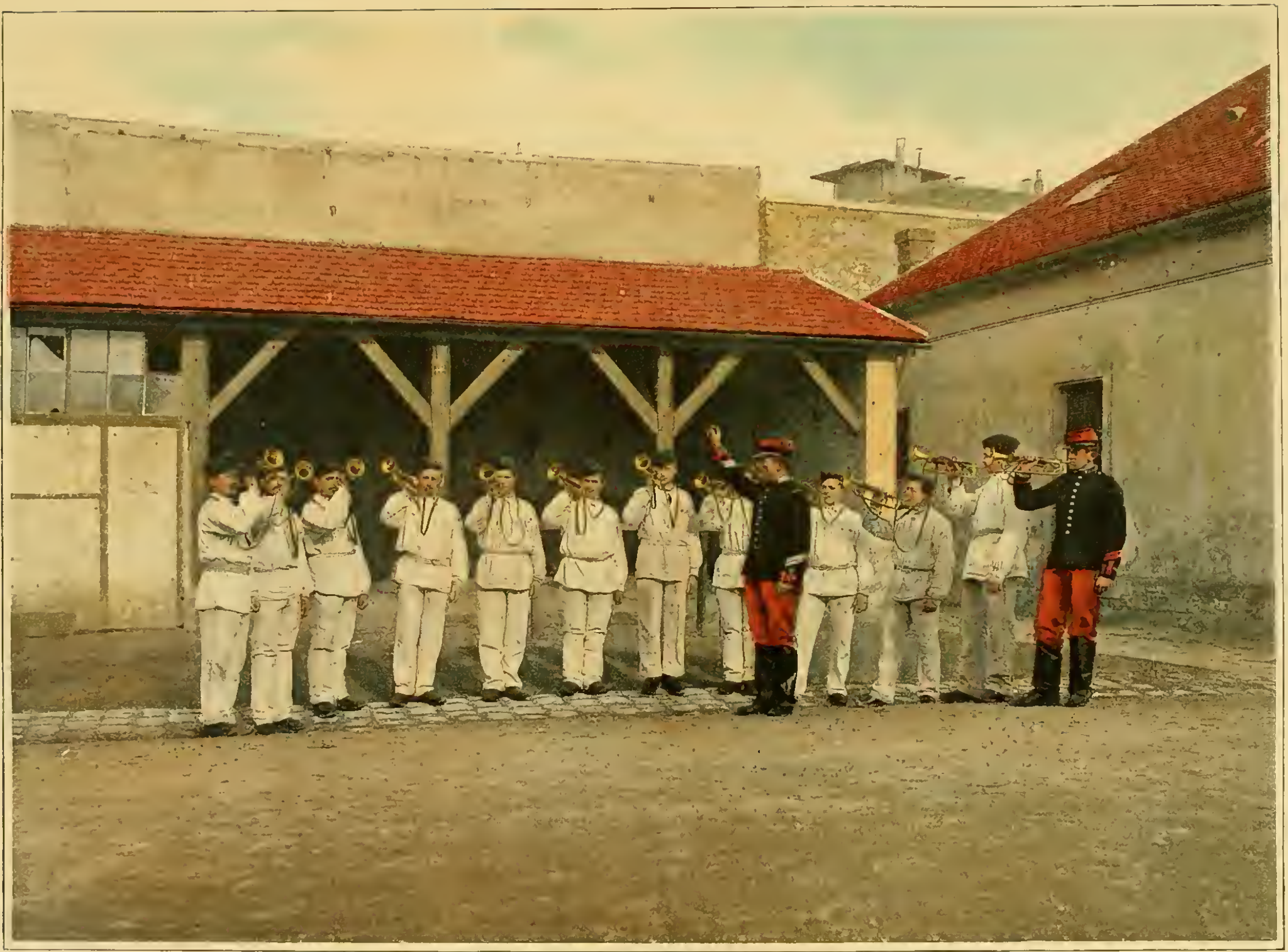

ÉGOLE DES TROMPETTES

Le maréchal des logis trompette major, secondé par le brigadier trompette, est chargé de l'instruction des trompettes et élèves trompcttes, auxqucls il apprend les différentes sonneries du service intérieur et du règlement d'exercices. Certains régiments de cavalerie forment, avec leurs trompettes, une fanfare qui égaie le régiment dans ses marches et joue dans les concerts publics en plein air des villes de garnison. 


\section{C:AVALERIE}

\section{Service Intérieur}

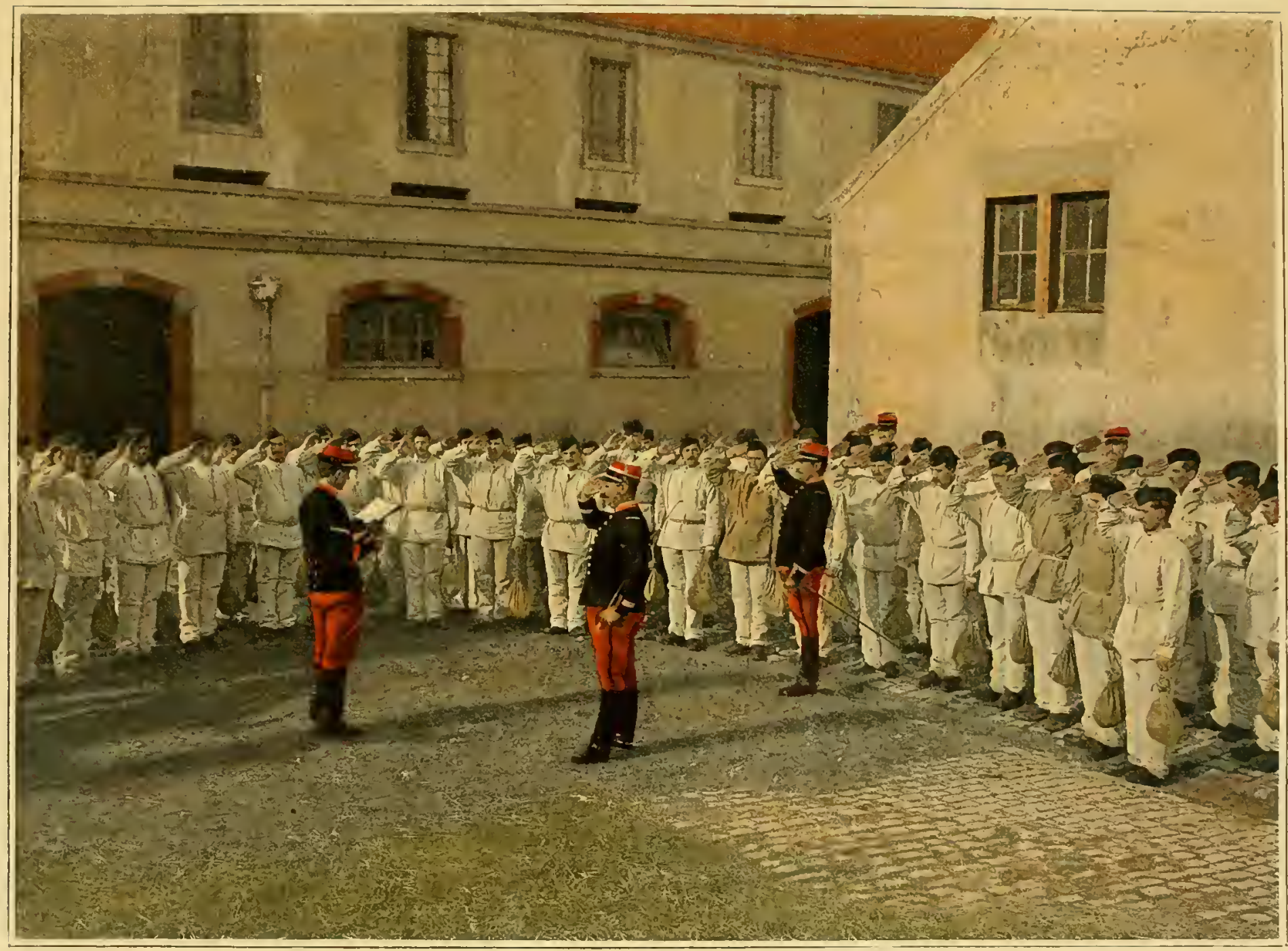

APPEL DU PANSAGE - LEGTURE DE LA DEGISION

L'escadron est réuni chacue jour, à l'heure fixéc, par le capitaine commandant, génćralement avant le pansage. Le marechal des logis.chet en tait l'appel on présence de lolticier de semaine qui lait donner lecture par le brigadier

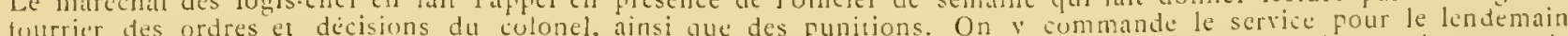

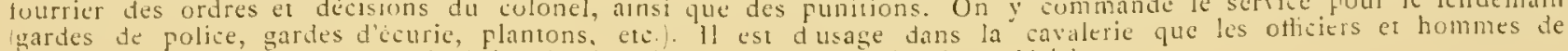
troupe saluent au moment oil le brigadier-fourrier commence il lire la "decision" 
G IVILERIE

Service Intérieur

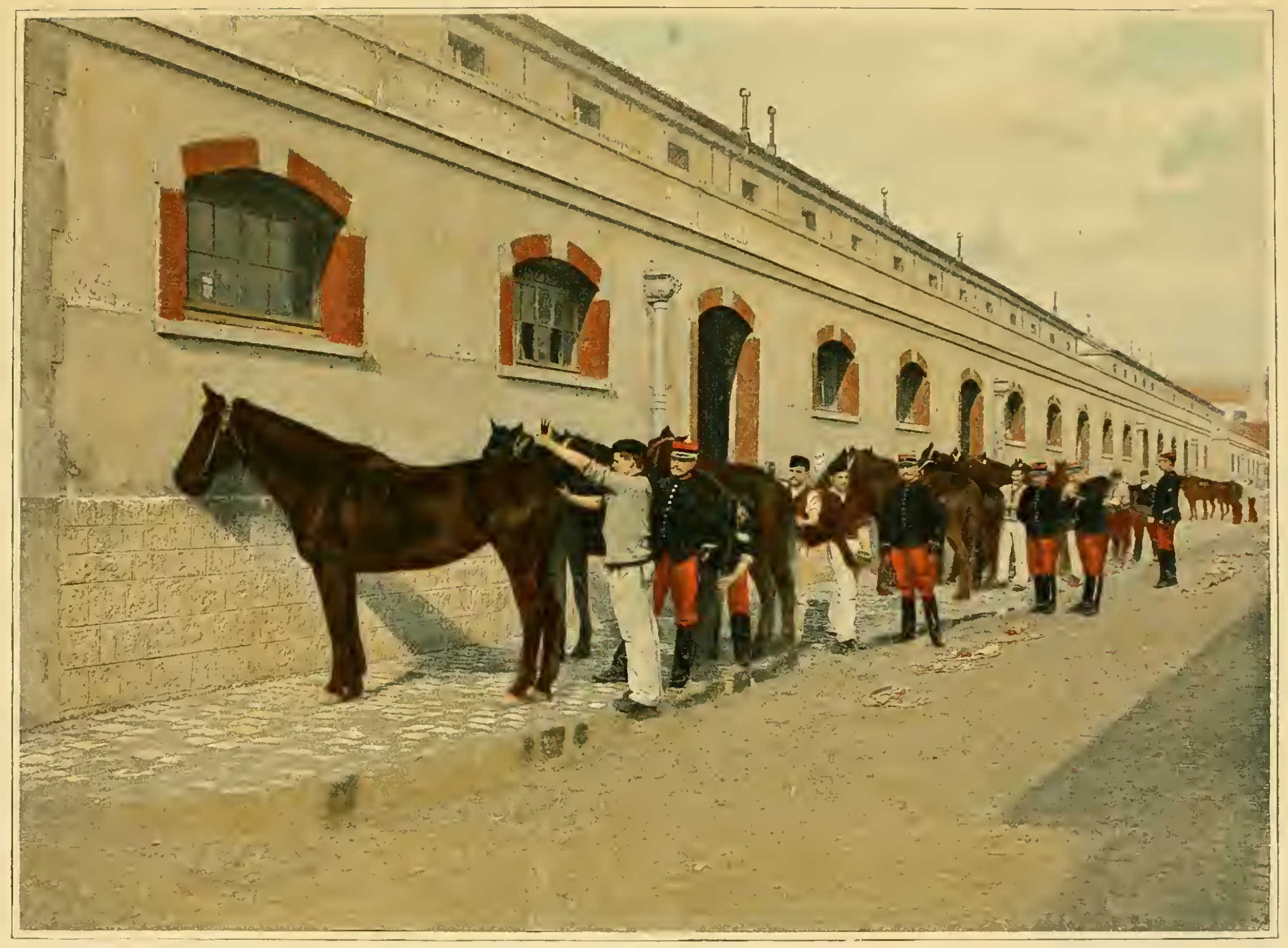

LE PANSAGE

"Faire le pansage" cos une des premieres choses que le cavalier apprend, dés sun arrivée au régiment.

Le pansage a licu au moins une fois par jour, autan que possible après le travail, et hors des écuries, toutes les fois que la température le permet. Il duit ètre exécuté avec une grande activité, en emplovant l’étrille, l’époussétte sorte de serviette de grosse toile, la brosse en crins, la brosse en chiendent et léponge. 
G $\perp$ VA LERIE

Service Intérieur

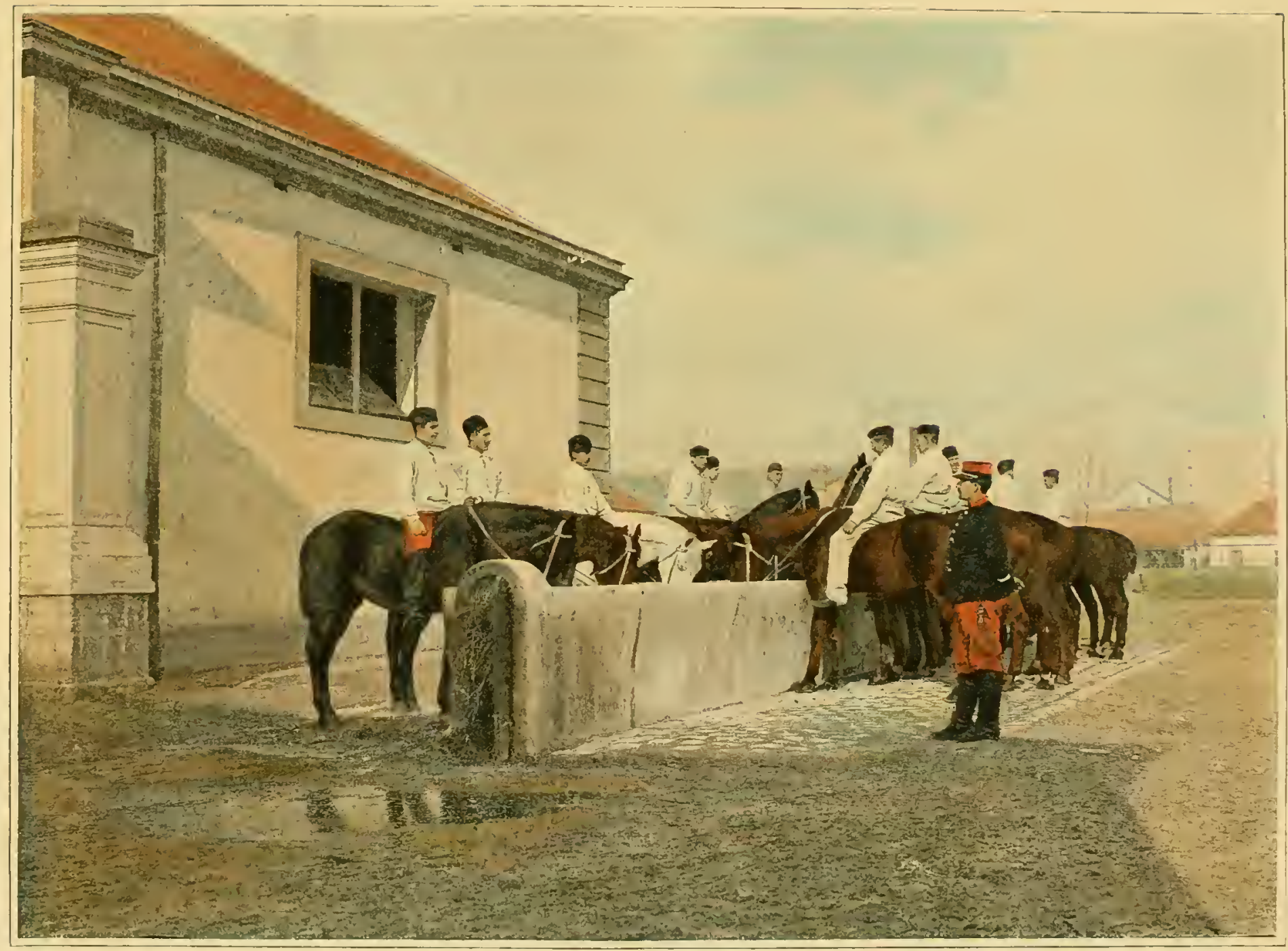

L'ABREUVOIR

Les chevaux doivent, en principe, boire deux fois par jour en été, et une fois en hiver. Il faut avoir soin de ne pas les conduire a l'abreuvoir quand ils on chaud, ni quand ils unt l'estomac plein. Il est bon, en tout cas, de toujours leur couper l'eau, c'est-à-dire de les empîcher de boire d'un seul trait. 
C.A I L L R I E

Service Intérieur

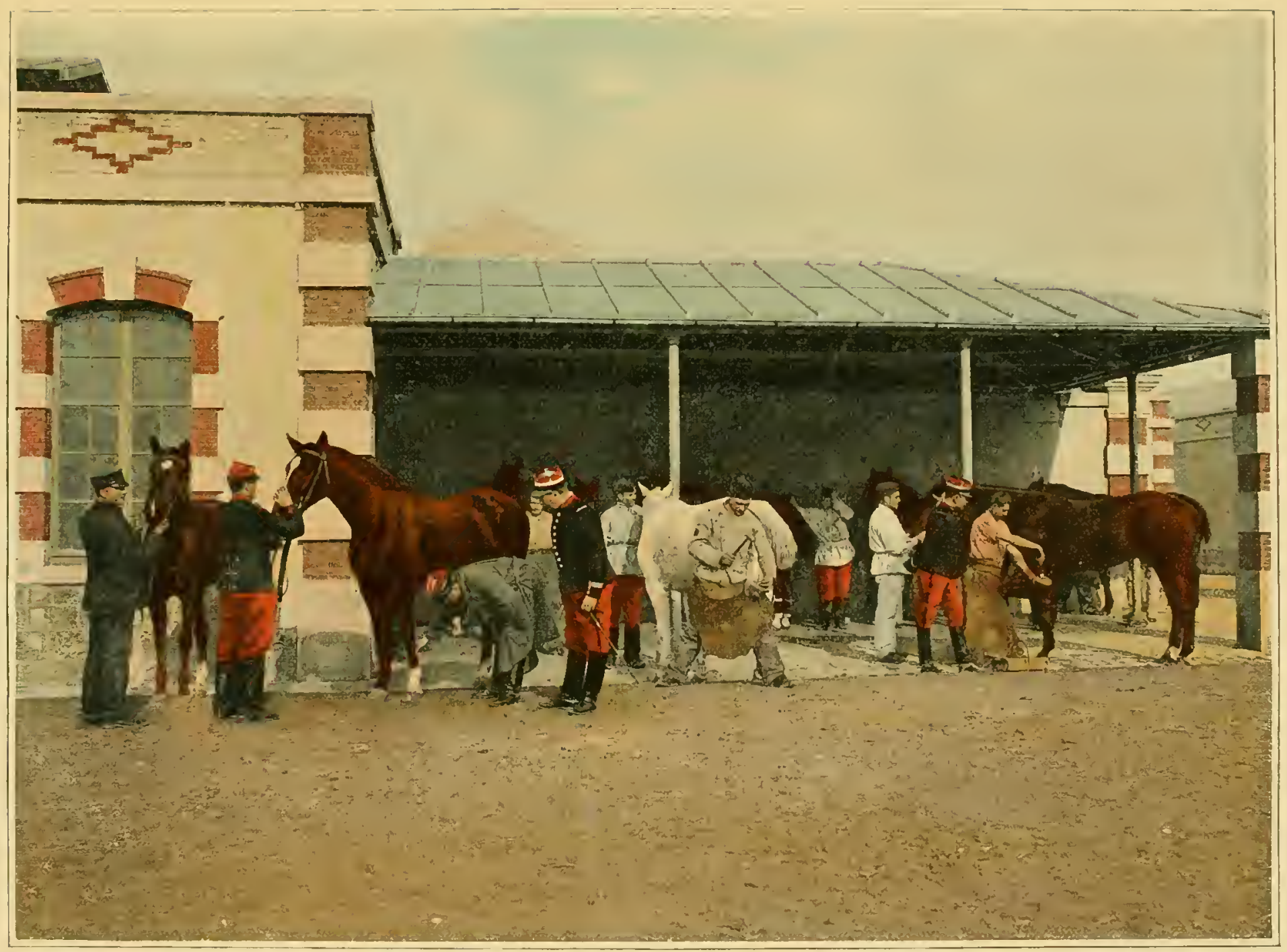

LA FORGE - VISITE DU VÉTÉRINAIRE

C'est avee les jambes de son cheval que marche le cavalier. Aussi, le ferrage des cheraux, exícuté par les maré. chaux du régiment, est-il l'objet des plus grands soins.

La visite des chevaux malades ou blessés cst passée chaque jour par le vétérinaire en fer qui les traite dans leur cscadron ou à l'intirmerie vétérinaire, et pratique toutes les opérations nécessaires four leur gućrison. 


\section{LiAlbum Militaire}

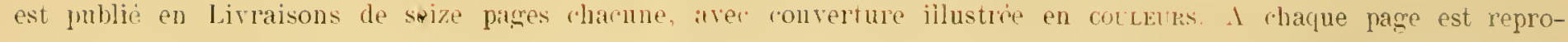

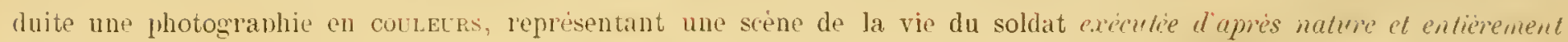
inidite.

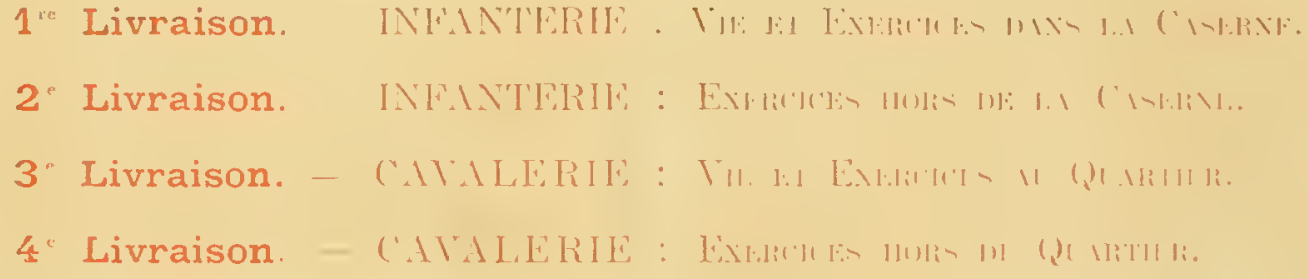

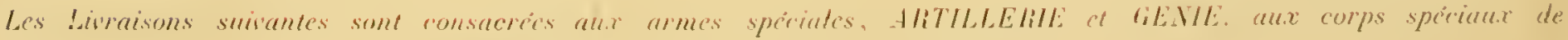

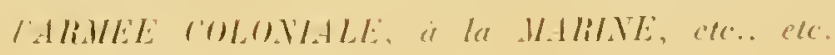

Les Livraisons seront mises en vente de mois en mois.

Reproduction interdite 